\title{
Модель взаимосвязи геометрии ветвей термоэлементов и показателей надежности при проектировании двухкаскадного охладителя в режиме $Q_{0 \max }$
}

\author{
Ю. И. Журавлев \\ Национальный университет «Одесская морская академия», ул. Дидрихсона, 8, г. Одесса, 65029, Украина
}

\begin{abstract}
Рассмотрено влияние геометрии ветвей термоэлементов в каскадах при $(l / S)_{1}=(l / S)_{2}$ на показатели надежности двухкаскадных термоэлектрических охлаждающих устройств для различных перепадов температуры $\Delta T=60 ; 70 ; 80 ; 90 \mathrm{~K}$ для отношения высоты термоэлемента к плошади $l / S=40 ; 20 ; 10 ; 4,5 ; 2,0$, тепловой нагрузке $Q_{0}=2,0$ Вт в режиме максимальной холодопроизводительности $Q_{0 \text { тах }}$ при последовательном электрическом соединении каскадов. Показано, что с уменьшением отношения $l / S$ в каскадах двухкаскадного термоэлектрического охладителя для различных перепадов температуры $\Delta T$ и фиксированной тепловой нагрузке $Q_{0}$ уменьшается интенсивность отказов $\lambda$, а, следовательно, увеличивается вероятность безотказной работы $P$.
\end{abstract}

Ключевые слова: Термоэлементы; Холодопроизводительность; Интенсивность отказов

\section{Модель взаємозв'язку геометрії гілок термоелементів і показників надійності при проектуванні двокаскадного охолоджувача в режимі $Q_{0 \max }$}

\section{Ю. І. Журавльов}

Національний університет «Одеська морська академія», вул. Дідріхсона, 8, м. Одеса, 65029, Україна

\begin{abstract}
Розглянуто вплив геометрії гілок термоелементів в каскадах $(l / S)_{1}=(l / S)_{2}$ на показники надійності двокаскадних термоелектричних охолоджуючих пристроїв для різних перепадів температури $\Delta T=60 ; 70 ; 80 ; 90 \mathrm{~K}$ для відносини висоти термоелемента до площі $l / S=40 ; 20 ; 10 ; 4,5 ; 2,0$, тепловому навантаженні $Q_{0}=2,0$ Bm в режимі максимальної холодопродуктивності $Q_{0 \text { тах }}$ при послідовному електричному з'єднанні каскадів. Показано, щчо зі зменшенням відносини $l / S$ в каскадах двокаскадного термоелектричного охолоджувача для різних перепадів температури $\Delta T$ та фіксованого теплового навантаження $Q_{0}$ зменшується інтенсивність відмов $\lambda$, а, отже, збільшується ймовірність безвідмовної роботи $P$.
\end{abstract}

Ключові слова: Термоелементи; Охолодження; Інтенсивність відмов

DOI: http://dx.doi.org/10.15673/ret.v53i2.599

(C) The Author(s) 2017. This article is an open access publication

This work is licensed under the Creative Commons Attribution 4.0 International License (CC BY)

http://creativecommons.org/licenses/by/4.0/

\section{1 Введение}

Термоэлектрические устройства относятся к твердотельным охладителям теплонагруженных компонентов радиоэлектронной аппаратуры. Их особенностями являются малые габариты, высокое быстродействие, отсутствие подвижных частей, что повышает надежность функционирования. Ужесточение требований к теплонагруженной аппаратуре приводит к необходимости повышения требований и к термоэлектрическим средствам обеспечения тепловых режимов, поскольку по модели надежности эти элементы соединены последовательно.
Вопросы повышения показателей надежности термоэлектрических охладителей (ТЭУ) детально рассмотрены относительно влияния тепловой нагрузки [1], режимов работы [2], технологии изготовления [3], материалов термочувствительных элементов [4]. Однако связь показателей надежности охладителей с конструктивными особенностями построения термоэлементов устройства не получила достаточного освещения. Поэтому анализ взаимосвязи конструктивных параметров термоэлемента с показателями надежности охладителя, как одной из составляющих эксплуатационной надежности устройства, является актуальной. 
При одинаковой геометрии ветвей термоэлементов в каскадах $(l / S)_{1}=(l / S)_{2}=$ const и одинаковой эффективности исходных материалов в каскадах можно повысить показатели надежности за счет выбора токового режима работы [5]. Возможно также повышение показателей надежности охладителей за счет повышения термоэлектрической эффективности материала термоэлементов. Однако на этапе проектирования термоэлектрических охладителей при использовании материала термоэлементов заданной термоэлектрической эффективностью имеется возможность выбора отношения $l / S$, изменение которого должно оказывать влияние и на показатели надежности двухкаскадного термоэлектрического охладителя.

Целью работы является определение возможности повышения показателей надежности термоэлектрического охладителя конструктивными методами за счет вариации геометрии ветвей термоэлементов.

В проведенных исследованиях рассмотрено влияние геометрии ветвей термоэлементов в каскадах при условии $(l / S)_{1}=(l / S)_{2}=40 ; 20 ; 10 ; 4,5 ; 2,0$ на основные параметры и показатели надежности при построении двухкаскадного ТЭУ для различных перепадов температуры $\Delta T=60 ; 70 ; 80 ; 90 \mathrm{~K}$ в режиме максимальной холодопроизводительности $Q_{0 \max }$.

\section{2 Модель взаимосвязи геометрии ветвей с показателями надежности}

Как известно, холодопроизводительность двухкаскадного ТЭУ определяется первым каскадом [6]:

$$
Q_{0}=n_{1} I_{\max 1}^{2} R_{1}\left(2 B_{1}-B_{1}^{2}-\Theta_{1}\right)=n_{1} \gamma_{1}\left(2 B_{1}-B_{1}^{2}-\Theta_{1}\right),
$$

где $n_{1}-$ количество термоэлементов в первом каскаде, шт.;

$I_{\max }=\bar{e} T_{0} / R_{1}-$ максимальный рабочий ток первого каскада, А;

$$
R=\frac{l}{\bar{\sigma} S}-\text { электрическое сопротивление первого }
$$
каскада, Ом;

$\bar{e}, \bar{\sigma}-$ усредненные значения коэффициента термоЭДС, В/К, и электропроводности, См/см, ветви термоэлемента соответственно;

$T_{0}$ - температура теплопоглощающего спая, К;

$B_{1}$ - относительный рабочий ток первого каскада, $B_{1}$ $=I / I_{\max 1}$, отн. ед.;

I - величина рабочего тока, А;

$\Theta_{1}$ - относительный перепад температуры первого каскада, $\Theta_{1}=\Delta T_{1} / \Delta T_{\max 1}=\left(T_{1}-T_{0}\right) / \Delta T_{\max 1}$;

$T_{1}$ - промежуточная температура, $\mathrm{K}$;

$\Delta T_{\max 1}$ - максимальный перепад температуры первого каскада, $\mathrm{K}, \Delta T_{\max 1}=0,5 \bar{z}_{1} T_{0}^{2}$;

$\bar{Z}_{1}$ - усредненное значение эффективности термоэлектрического материала первого каскада, $1 / \mathrm{K}$;

$\gamma_{1}$ - максимальная термоэлектрическая мощность охлаждения первого каскада,

$$
\gamma=I_{\max 1}^{2} R_{1}=\bar{e}_{1}^{2} \bar{\sigma}_{1} T_{0}^{2}(S / l)_{1}, \text { Вт. }
$$

При последовательном электрическом соединении каскадов

$$
I_{\max 1} B_{1}=I_{\max 2} B_{2}=I,
$$

где $I_{\max 2}$ - максимальный рабочий ток второго каскада, $I_{\max 2}=\bar{e}_{2} T_{1} / R_{2}, \mathrm{~A}$;

$R_{2}$ - электрическое сопротивление ветви термоэлемента второго каскада, $R_{2}=\frac{l_{2}}{\bar{\sigma}_{2} S_{2}}$, Ом;

$(l / S)_{2}$ - отношение высоты $l_{2}$ к площади поперечного сечения $S_{2}$ ветви термоэлемента второго каскада, $1 / \mathrm{cm;}$

$\bar{e}_{2}, \bar{\sigma}_{2}-$ усредненные значения коэффициента термо-ЭДС, В/К, и электропроводности, См/см, ветви термоэлемента второго каскада;

$B_{2}$ - относительный рабочий ток второго каскада, $B_{2}$ $=I / I_{\max 2}$;

Отношение количества термоэлементов в смежных каскадах можно записать в виде

$$
n_{2} / n_{1}=\frac{I_{\max 1}^{2} R_{1}}{I_{\max 2}^{2} R_{2}} \frac{2 B_{1}\left(1+\frac{\Delta T_{\max }}{T_{0}} \Theta_{1}\right)+B_{1}^{2}-\Theta_{1}}{2 B_{2}-B_{2}^{2}-\Theta_{2}},
$$

где $\Theta_{2}=\frac{\Delta T_{2}}{\Delta T_{\max 2}}=\frac{T-T_{1}}{0,5 \bar{z}_{2} T_{1}^{2}}-$ относительный перепад температуры во втором каскаде, отн. ед.;

$T$ - температура тепловыделяющего спая, К;

$\Delta T_{\max 2}$ - максимальный перепад температуры второго каскада, $\Delta T_{\max 2}=0,5 \bar{z}_{2} T_{1}^{2}, \mathrm{~K}$;

$\bar{z}_{2}$ - усредненное значение эффективности термоэлектрического материала второго каскада, $1 / K$;

Мощность потребления первого и второго каскадов, соответственно, можно записать в виде

$$
\begin{aligned}
& W_{1}=2 n_{1} I_{\max 1}^{2} R_{1} B_{1}\left(B_{1}+\frac{\Delta T_{\max 1}}{T_{0}} \Theta_{1}\right) ; \\
& W_{2}=2 n_{2} I_{\max 2}^{2} R_{2} B_{2}\left(B_{2}+\frac{\Delta T_{\max 2}}{T_{1}} \Theta_{2}\right) .
\end{aligned}
$$

Общая мощность потребления двухкаскадного ТЭУ составляет

$$
W_{\Sigma}=W_{1}+W_{2}=\sum_{i=1}^{N} W_{i}
$$

Холодильный коэффициент $E^{N=2}$ двухкаскадного ТЭУ можно записать как

$$
E^{N=2}=Q_{0} / W_{\Sigma} .
$$

Падение напряжения на двухкаскадном ТЭУ

$$
U_{\Sigma}=W_{\Sigma} / I
$$

Относительную величину интенсивности отказов $\lambda / \lambda_{0}$ двухкаскадного ТЭУ можно представить в виде суммы интенсивностей отказов каскадов: 


$$
\lambda / \lambda_{0}=n_{1} B_{1}^{2}\left(\theta_{1}+C_{1}\right) \frac{\left(B_{1}+\frac{\Delta T_{\max 1}}{T_{0}} \Theta_{1}\right)^{2}}{\left(1+\frac{\Delta T_{\max 1}}{T_{0}} \Theta_{1}\right)^{2}} K_{T 1}+n_{2} B_{2}^{2}\left(\theta_{2}+C_{2}\right) \frac{\left(B_{2}+\frac{\Delta T_{\max 2}}{T_{1}} \Theta_{2}\right)^{2}}{\left(1+\frac{\Delta T_{\max 2}}{T_{1}} \Theta_{2}\right)^{2}} K_{T 1},
$$

где $C_{1}$ и $C_{2}$ - относительная величина тепловой нагрузки первого и второго каскадов соответственно, $C_{1}=Q_{0} /\left(n_{1} I_{\max 1}^{2} R_{1}\right), C_{2}=\left(Q_{1}+W_{1}\right) /\left(n_{2} I_{\text {max } 2}^{2} R_{2}\right)$;

$K_{T 1}$ и $K_{T 2}-$ коэффициент значимости, зависящий от температуры [6];

$\lambda_{0}-$ номинальная интенсивность отказов, $\lambda_{0}=3 \cdot 10^{-8}, 1 /$ ч.

Вероятность безотказной работы $P$ двухкаскадного ТЭУ можно представить в виде:

$$
P=\exp (-\lambda t)
$$

где $t-$ назначенный ресурс, $t=10^{4}$, ч.

Результаты расчетов основных параметров и показателей надежности двухкаскадного ТЭУ при $(l / S)_{1}=(l / S)_{2}=40 ; 20 ; 10 ; 4,5 ; 2,0$, при перепадах температуры $\Delta T=60 ; 70 ; 80 ; 90$ К при тепловой нагрузке $Q_{0}=2,0$ Вт в режиме $Q_{0 \max }$ приведены в таблице 1.

Tаблица 1 - Основные параметры и показатели надежности двухкаскадного ТЭУ при $(l / S)_{1}=(l / S)_{2}=v a r, T=300$

\begin{tabular}{|c|c|c|c|c|c|c|c|c|c|c|c|c|c|c|c|c|}
\hline $\begin{array}{l}(l / S)_{1} \\
= \\
(l / S)_{2}\end{array}$ & $\begin{array}{c}R_{1} \cdot 1^{3}, \\
\text { Ом }\end{array}$ & $\begin{array}{c}R_{2} \cdot \mathbf{1 0}^{3} \\
\text { ОM }\end{array}$ & $\underset{\mathrm{A}}{I_{\max 1},}$ & $\underset{\mathrm{A}}{I_{\max 2}}$ & $\begin{array}{l}\text { I, } \\
\text { A }\end{array}$ & $\begin{array}{l}n_{1}, \\
\text { سIT }\end{array}$ & $\begin{array}{l}n_{2}, \\
\text { шт }\end{array}$ & $\begin{array}{c}n_{1}+n_{2} \\
\text { IIIT }\end{array}$ & $U_{1}, \mathrm{~B}$ & $U_{2}, \mathbf{B}$ & $U_{\Sigma}, \mathbf{B}$ & $\begin{array}{c}C_{1}, \\
\text { отн. ед. }\end{array}$ & $\begin{array}{c}C_{2}, \\
\text { отн. ед. }\end{array}$ & $\lambda / \lambda_{0}$ & $\begin{array}{c}\lambda \cdot 10^{8} \\
1 / \mathbf{4}\end{array}$ & $\boldsymbol{P}$ \\
\hline \multicolumn{17}{|c|}{$\begin{array}{l}\Delta T=60 \mathrm{~K} \\
65 ; \Theta_{1}=0,426 ; \Theta_{2}=0,389 ; K_{T 1}=1,035 ; K_{T 2}=1,018 \\
6 \mathrm{BT} ; W_{\Sigma}=41,4 \mathrm{BT} ; E=0,0483 ; n_{2} / n_{1}=3,65\end{array}$} \\
\hline 40 & 36,4 & 42,6 & 1,19 & 1,23 & 1,19 & 68,2 & 249,0 & 317,2 & 6,52 & 28,2 & \begin{tabular}{|l|}
34,8 \\
\end{tabular} & \multirow{4}{*}{0,574} & \multirow{4}{*}{0,61} & 292,0 & 875,0 & 0,9162 \\
\hline 20 & 18,2 & 21,3 & 2,37 & 2,37 & 2,37 & 34,1 & 124,4 & 158,5 & 3,27 & 14,2 & 17,5 & & & 145,9 & 437,6 & 0,9572 \\
\hline 10 & 9,09 & 10,64 & 4,75 & 4,75 & 4,75 & 17,0 & 62,0 & 79,0 & 1,63 & 7,10 & 8,7 & & & 72,7 & 218,2 & 0,9784 \\
\hline 4,5 & 4,09 & 4,79 & 10,6 & 10,93 & 10,56 & 7,6 & 27,9 & 35,5 & 0,73 & 3,18 & 3,90 & & & 32,8 & 98,3 & 0,9902 \\
\hline 2,0 & 1,82 & 2,13 & 23,7 & 24,6 & 23,7 & 2,3 & 12,4 & 15,8 & 0,33 & 1,41 & 1,74 & 0,575 & 0,57 & 14,0 & 42,0 & 0,9958 \\
\hline \multicolumn{17}{|c|}{$\begin{array}{c}\Delta T=70 \mathrm{~K} \\
T_{1}=262 \mathrm{~K} ; B_{1}=1,0 ; B_{2}=0,958 ; \Theta_{1}=0,562 ; \Theta_{2}=0,469 ; K_{T 1}=1,043 ; K_{T 2}=1,021 \\
W_{1}=10,4 \mathrm{BT} ; W_{2}=49,5 \mathrm{BT} ; W_{\Sigma}=60,0 \mathrm{BT} ; E=0,0333 ; n_{2} / n_{1}=3,90\end{array}$} \\
\hline 40 & 36,8 & 42,1 & 1,17 & 1,22 & 1,17 & 96,6 & 376,8 & 473,4 & 8,92 & 42,4 & \begin{tabular}{|l|}
51,3 \\
\end{tabular} & \multirow{5}{*}{0,44} & \multirow{5}{*}{0,52} & 487,8 & 1283 & 0,8796 \\
\hline 20 & 17,4 & 21,05 & 2,33 & 2,43 & 2,33 & 48,3 & 188,4 & 236,7 & 4,46 & 21,2 & 25,7 & & & 213,9 & 641,5 & 0,9379 \\
\hline 10 & 8,7 & 10,53 & 4,65 & 4,85 & 4,65 & 24,3 & 94,7 & 119,0 & 2,24 & 10,65 & 12,9 & & & 107,7 & 323,0 & 0,9682 \\
\hline 4,5 & 3,91 & 4,74 & 10,35 & 10,78 & 10,36 & 10,9 & 42,5 & 53,4 & 1,0 & \begin{tabular}{|l|}
4,78 \\
\end{tabular} & 5,80 & & & 48,4 & 145,2 & 0,9856 \\
\hline 2,0 & 1,74 & 2,10 & 23,3 & 24,3 & 23,3 & 4,8 & 18,8 & 23,6 & 0,48 & 2,10 & \begin{tabular}{|l|}
2,56 \\
\end{tabular} & & & 21,5 & 64,5 & 0,9936 \\
\hline \multicolumn{17}{|c|}{$\begin{array}{c}\Delta T=80 \mathrm{~K} \\
T_{1}=256 \mathrm{~K} ; B_{1}=1,0 ; B_{2}=0,941 ; \Theta_{1}=0,716 ; \Theta_{2}=0,571 ; K_{T 1}=1,052 ; K_{T 2}=1,024 ; \\
W_{1}=16,5 \mathrm{BT} ; W_{2}=90,9 \mathrm{BT} ; W_{\Sigma}=107,5 \mathrm{BT} ; E=0,0186 ; n_{2} / n_{1}=4,34\end{array}$} \\
\hline 40 & 33,4 & 41,6 & 1,12 & 1,19 & 1,12 & 169,2 & 734,0 & \begin{tabular}{|l|}
903,2 \\
\end{tabular} & 14,8 & 81,2 & \begin{tabular}{|l|}
96,0 \\
\end{tabular} & \multirow{5}{*}{0,28} & \multirow{5}{*}{0,43} & 776 & 2328 & 0,7923 \\
\hline 20 & 16,7 & 20,8 & 2,24 & 2,38 & 2,24 & 84,6 & 367,0 & 451,4 & 7,4 & 40,6 & 48,0 & & & 388 & 1165 & 0,8900 \\
\hline 10 & 8,33 & 10,42 & 4,49 & 4,77 & 4,49 & 41,9 & 182,0 & 223,9 & 3,68 & 20,25 & 23,9 & & & 192,9 & \begin{tabular}{|l}
578,7 \\
\end{tabular} & 0,9438 \\
\hline 4,5 & 3,75 & 4,69 & 9,97 & 10,6 & 9,97 & 18,9 & 81,9 & 100,8 & 1,66 & 9,12 & 10,8 & & & 86,8 & 260,5 & 0,9743 \\
\hline 2,0 & 1,67 & 2,10 & 22,4 & 23,6 & 22,4 & 8,4 & 36,5 & 44,9 & 0,74 & \begin{tabular}{|l|}
4,06 \\
\end{tabular} & 4,8 & & & 39,7 & 119,0 & 0,9882 \\
\hline \multicolumn{17}{|c|}{$\begin{array}{c}\Delta T=90 \mathrm{~K} \\
T_{1}=250 \mathrm{~K} ; B_{1}=1,0 ; B_{2}=0,914 ; \Theta_{1}=0,907 ; \Theta_{2}=0,684 ; K_{T 1}=1,062 ; K_{T 2}=1,028 \\
W_{1}=51,2 \mathrm{BT} ; W_{2}=350,6 \mathrm{BT} ; W_{\Sigma}=401,8 \mathrm{BT} ; E=0,0050 ; n_{2} / n_{1}=5,25\end{array}$} \\
\hline 40 & 32,0 & 40,8 & 10,8 & 1,18 & 1,08 & 582 & 3054 & \begin{tabular}{|l|}
3636 \\
\end{tabular} & 47,6 & \begin{tabular}{|l|}
326 \\
\end{tabular} & 373,6 & \multirow{5}{*}{0,093} & \multirow{5}{*}{0,31} & 2862 & 8586 & 0,4238 \\
\hline 20 & 16,0 & 20,41 & 2,35 & 2,35 & 2,15 & 291 & 1527 & 1818 & 23,8 & \begin{tabular}{|l|}
163,0 \\
\end{tabular} & 186,8 & & & 1431 & 4293 & 0,651 \\
\hline 10 & 8,0 & 10,2 & 4,31 & 4,71 & 4,31 & 145,4 & 763,3 & 908,7 & \begin{tabular}{|l|}
11,9 \\
\end{tabular} & 81,5 & 93,4 & & & 715 & 2146 & 0,8069 \\
\hline 4,5 & 3,6 & 4,59 & 9,57 & 10,46 & 9,57 & 65,2 & 342,0 & 407,0 & 5,35 & 36,6 & 42,0 & & & 320,9 & 962,7 & 0,9082 \\
\hline 2,0 & 1,6 & 2,04 & 21,5 & 23,5 & 21,5 & 29,1 & 152,7 & 181,8 & 2,38 & 16,3 & 18,7 & & & 144 & 432 & 0,9577 \\
\hline
\end{tabular}
К, $Q_{0}=2,0$ Вт для различных значений $\Delta T$ в режиме $Q_{0 \max }$

\section{3 Анализ результатов моделирования двух- каскадного ТЭУ}

Анализ результатов расчета основных параметров и показателей надежности двухкаскадного ТЭУ, приве- денных в таблице, показывает, что с уменьшением отношения $l / S$ при заданном перепаде температуры $\Delta T$ и тепловой нагрузке $Q_{0}$ :

- увеличивается величина максимального рабочего тока $I_{\max 1}$ и $I_{\max 1}$ в каскадах; 
- уменьшается электрическое сопротивление ветвей термоэлементов в каскадах $R_{1}$ и $R_{2}$;

- увеличивается величина рабочего тока $I$;

- уменьшается суммарное количество термоэлементов $\left(n_{1}+n_{2}\right)$, например при $\Delta T=70 \mathrm{~K}$ уменьшение отношения $l / S$ в каскадах от 20 до 2 приводит к уменьшению суммарного количества термоэлементов в 10 раз;

- уменьшается общее падение напряжения $U_{\Sigma}$;

- уменьшается интенсивность отказов $\lambda / \lambda_{0}$ и, соответственно, увеличивается вероятность безотказной работы $P$, например при $\Delta T=70 \mathrm{~K}$, уменьшая отношение $l / S$ в каскадах от 20 до 2, можно уменьшить интенсивность отказов на порядок, тем самым повысить, и довольно значительно, вероятность безотказной работы $P$.

Промежуточная температура $T_{1}$, относительные перепады температуры в каскадах $\Theta_{1}$ и $\Theta_{2}$, относительные рабочие токи в каскадах $B_{1}$ и $B_{2}$, отношение количества термоэлементов в смежных каскадах $n_{2} / n_{1}$, общая потребляемая мощность $W$, холодильный $n_{2} / n_{1}$ коэффициент $E$ не зависят от геометрии ветвей термоэлементов при заданном перепаде температуры $\Delta T$.

Из приведенных на рисунках 1-5 данных видно, что с ростом перепада температуры $\Delta T$ для различных значений отношения $l / S$ при заданной тепловой нагрузке $Q_{0}=2,0$ Вт:

- уменьшается промежуточная температура $T_{1}$ (рисунок 1, поз. 1);

- уменьшается холодильный коэффициент $E$ (рисунок 1, поз. 2);

- увеличивается отношение количества термоэлементов в смежных каскадах (рисунок 1, поз. 3);

- уменьшается величина рабочего тока I (рисуНок 2);

- увеличивается суммарное количество термоэлементов $\left(n_{1}+n_{2}\right)$ (рисунок 3$)$;

- увеличивается интенсивность отказов $\lambda / \lambda_{0}$ (рисунок 4);

- уменьшается вероятность безотказной работы $P$ (рисунок 5).

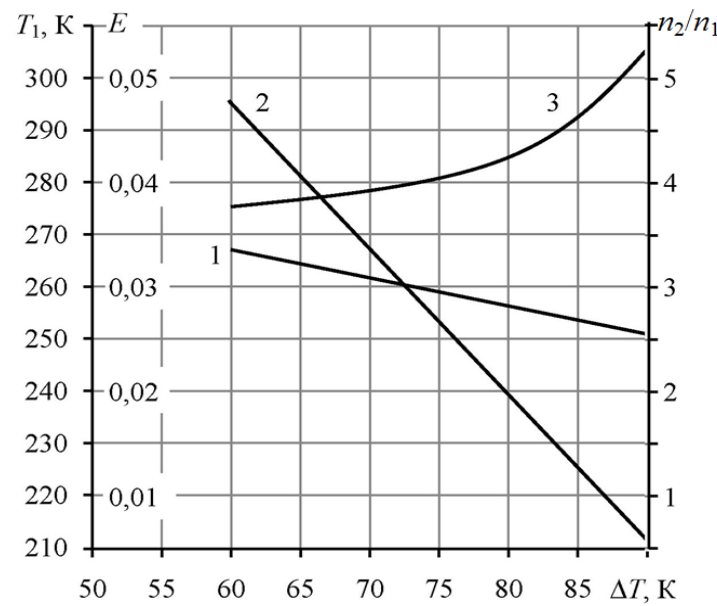

Рисунок 1 - Зависимость промежуточной температуpы $T_{1}(1)$, холодильного коэффициента $E$ (2) и отношения $n_{2} / n_{1}$ (3) двухкаскадного охладителя от перепада температуры $\Delta T$ при $T=300 \mathrm{~K} ; Q_{0}=2,0 \mathrm{Bm}$ в режиме максимальной холодопроизводительности $Q_{\text {отах }}$

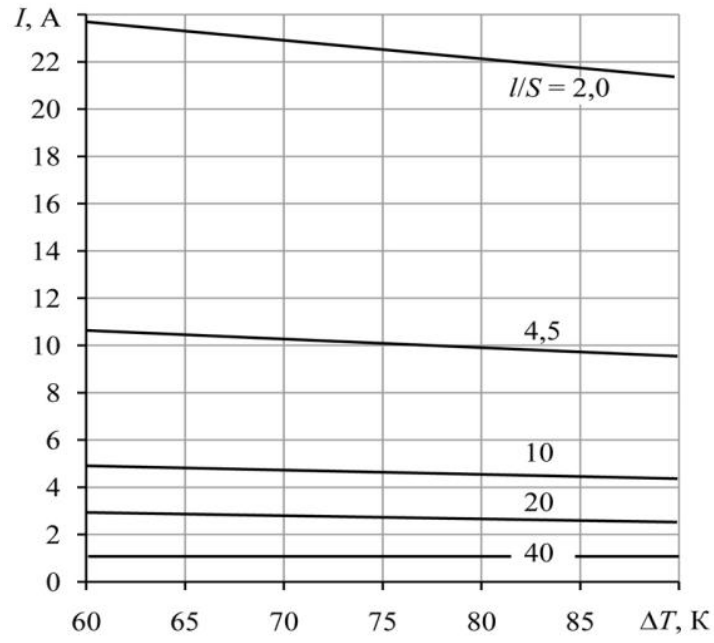

Рисунок 2 - Зависимость рабочего тока I двухкаскадного термоэлектрического охладителя от перепада температуры $\Delta T$ для различных значений отношения $\mathrm{l} / \mathrm{s}$ при $T=300 \mathrm{~K} ; Q_{0}=2,0 \mathrm{Bm}$ в режиме максимальной холодопроизводительности $Q_{\text {отах }}$

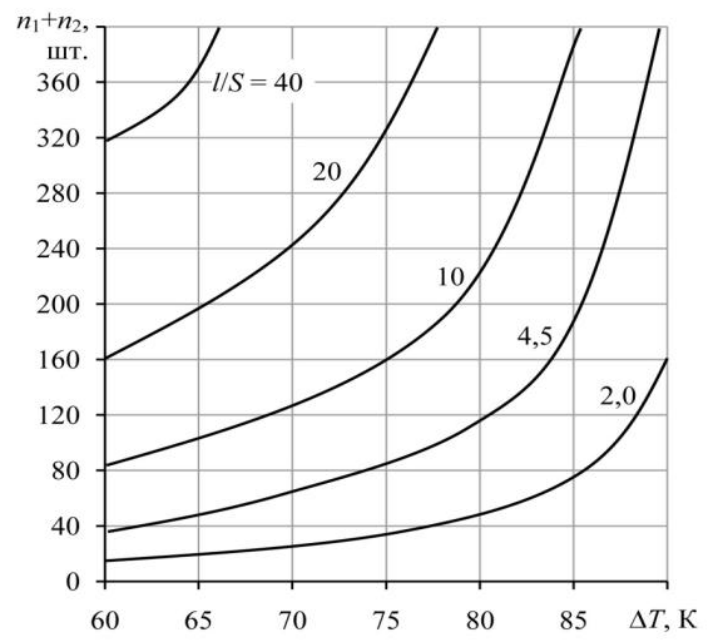

Рисунок 3 - Зависимость суммарного количества термоэлементов двухкаскадного термоэлектрического охладителя от перепада температуры $\Delta T$ для различных значений отношения $\mathrm{l} / \mathrm{s}$ при $T=300 \mathrm{~K} ; Q_{0}=2,0 \mathrm{Bm} в$ режиме максимальной холодопроизводительности $Q_{0 \text { тах }}$

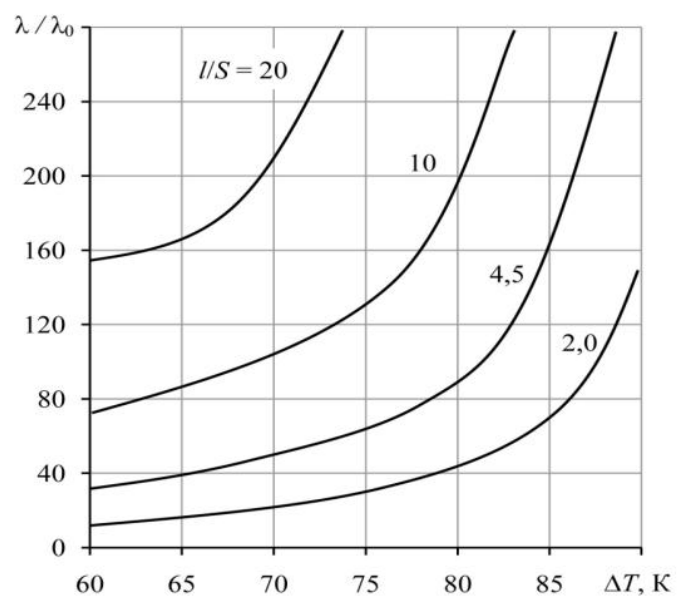

Рисунок 4 - Зависимость относительной интенсивности отказов $\lambda / \lambda_{0}$ двухкаскадного охладителя от перепада температуры $\Delta T$ для различных значений отношения $\mathrm{l} / \mathrm{s}$ при $\mathrm{T}=300 \mathrm{~K} ; Q_{0}=2,0 \mathrm{Bm} ; \lambda_{0}=3 \cdot 10^{-8} 1 /$ ч в режиме максимальной холодопроизводительности $Q_{0 \text { тах }}$ 


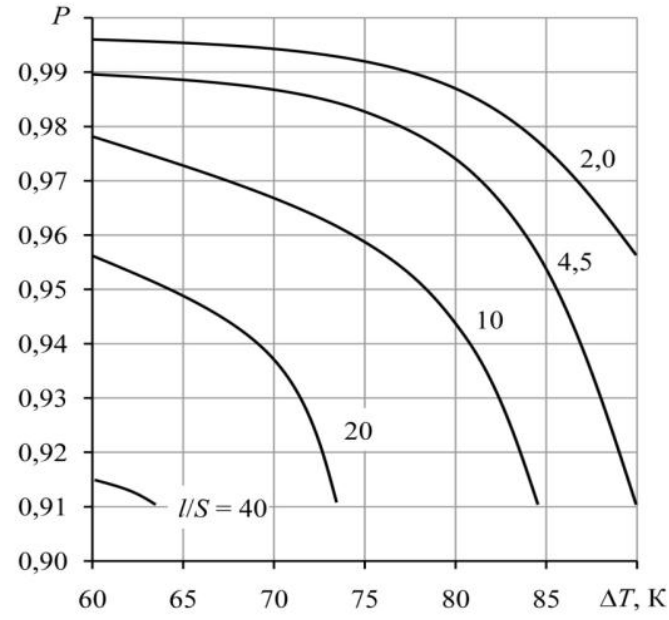

Рисунок 5 - Зависимость вероятности безотказной работь $P$ двухкаскадного охладителя от перепада температуры $\Delta T$ для различных значений отношения $l / s$ при $T=300 \mathrm{~K} ; Q_{0}=2,0 \mathrm{Bm} ; t=10^{4}$ ч в режиме $Q_{\text {отах }}$

Приведенные графические зависимости позволяют конструктору наглядно оценить степень влияния перепада температуры на основные показатели двухкаскадного термоэлектрического охладителя.

\section{4 Выводы}

1. Разработана модель взаимосвязи геометрии вервей термоэлементов с надежностными и энергетическими показателями двухкаскадного охладителя.

2. Показано, что с уменьшением отношения $l / S$ в каскадах с одинаковой геометрией ветвей термоэлемен тов можно на порядок уменьшить интенсивность отказов двухкаскадного ТЭУ для различных перепадов температуры $\Delta T$ и фиксированной тепловой нагрузке $Q_{0}$ в режиме $Q_{0 \max }$.

\section{Литература}

1. Zebarjadi, M., Esfarjani K., Dresselhaus, M. S., Ren, Z. F., Chen, G. (2012). Perspectives on thermoelectrics: from fundamentals to device applications. Energy \& Environmental Science, 5, 5147-5162.

2. Ping Yang (2010). Approach on thermoelectricity reliability of board-level backplane based on the orthogonal experiment design. International Journal of Materials and Structural Integrity, 4(2-4), 170-185.

3. Singh R. (2008). Experimental Characterization of Thin Film Thermoelectric Materials and Film Deposition VIA Molecular Beam Epitaxy. University of California, 54.

4. Sootsman J. R., Chung D. Y., Kanatzidis M. G. (2009). New and Old Concepts in Thermoelectric Materials. Angewandte Chemie International Edition, 48 (46), 8616-8639.

5. Зайков В. П., Мещеряков В. И., Журавлев Ю. И. Прогнозирование показателей надежности термоэлектрических охлаждающих устройств. Книга 2. Каскадные устройства. - Одесса: Политехпериодика, 2016. $124 \mathrm{c}$.

6. Зайков В. П., Киншова Л. А., Моисеев В. Ф. Прогнозирование показателей надежности термоэлектрических охлаждающих устройств. Книга 1. Однокаскадные устройства. - Одесса: Политехпериодика, 2009. - 120 с.

Отримана в редакції 20.01.2017, прийнята до друку 25.04.2017

\title{
Correlation Model of the Thermal Elements Branches Geometry and Reli- ability Indicators for Two-Stage Cooler Design in the $Q_{0 \text { Max }}$ Mode
}

\section{Yu. Zhuravlov}

National University «Odessa Maritime Academy», 8 Didrikhsona str., Odessa, 65029, Ukraine

\begin{abstract}
Influence of the thermoelements branches geometry in cascades at $(l / S)_{1}=(l / S)_{2}$ on the reliability parameters of two-stage thermoelectric cooling devices for various temperature drops $\Delta T=60 ; 70 ; 80 ; 90 \mathrm{~K}$ for the ratio of the height of the thermoelement to the area $l / S=40 ; 20 ; 10 ; 4.5 ; 2.0$, the thermal load $Q_{0}=2.0 \mathrm{~W}$ in the maximum cooling capacity $Q_{0 \max }$ with a series of electrical cascades has been considered in the paper. A reliability-oriented model of a two-stage cooler has been developed that relates the relative intensity of failures to energy and design parameters. The effect of the ratio of the thermoelement length to the cross-sectional area on the working current, the total number of required thermoelements, the relative failure rate and the probability of failure-free operation of the device have been studied. It is shown that with a decrease in the $l / S$ ratio in cascades of a two-stage thermoelectric cooler for various temperature differences $\Delta T$ and a fixed thermal load $Q_{0}$, the failure rate $\lambda$ decreases, and, consequently, the probability of failure-free operation of $P$ increases.
\end{abstract}

Keywords: Thermoelements - Refrigeration capacity - Failure rate

\section{References}

1. Zebarjadi, M., Esfarjani K., Dresselhaus, M. S., Ren, Z.

F., Chen, G. (2012). Perspectives on thermoelectrics: from fundamentals to device applications. Energy \& Environmental Science, 5, 5147-5162.

2. Ping Yang (2010). Approach on thermoelectricity reliability of board-level backplane based on the ortho 
gonal experiment design. International Journal of Materials and Structural Integrity, 4(2-4), 170-185. DOI: https://doi.org/10.1504/ijmsi.2010.035205

3. Singh, R. (2008). Experimental Characterization of Thin Film Thermoelectric Materials and Film Deposition VIA Molecular Beam Epitaxy. University of California, 54. 4. Sootsman, J. R., Chung, D. Y., Kanatzidis, M. G. (2009). New and Old Concepts in Thermoelectric Materials. Angewandte Chemie International Edition, 48 (46), 8616-8639.

5. Zaikov, V.P., Meshcheryakov, V. I., Zhuravlev, Yu. I. (2016). Prediction of thermo-electric cooling devicesreliability indices. Book 2. Cascade devices. Odessa: Polytechperiodica, 124.

6. Zaykov, V. P., Kinshova, L. A., Moiseev, V. F. (2009). Prediction of reliability on thermoelectric cooling devices. KN.1 Single-stage devices. Odessa: Politehperiodika, 120.

Received 20 January 2017 Approved 25 April 2017 Available in Internet 29 May 2017 